\title{
TAFONOMÍA DE LOS CARBONATOS TEMPLADOS PLIOCENOS DE LA CUENCA DE ALMAYATE (MÁLAGA, S DE ESPAÑA)
}

\author{
Julio AGUIRRE ${ }^{1}$ y Nahum MÉNDEZ-CHAZARRA \\ Dpto. de Estratigrafía y Paleontología, Facultad de Ciencias, Fuentenueva s/n, \\ Universidad de Granada, 18002, Granada \\ ${ }^{1}$ E-mail: jaguirre@ugr.es
}

Aguirre, J. \& Méndez-Chazarra, N. 2010. Tafonomía de los carbonatos templados pliocenos de la Cuenca de Almayate (Málaga, S de España). [Taphonomy of temperate carbonate pliocene deposits of the Almayate basin (Málaga, S. Spain).] Revista Española de Paleontología, 25 (2), 149-163. ISSN 0213-6937.

\begin{abstract}
Taphonomic analyses of Pliocene fossil assemblages of the Almayate Basin (Málaga, S Spain) have been carried out. The study was performed quantifying different taphonomic attributes. Values were used to make a Qmode cluster analysis to infer taphofacies models. Results suggest high energy conditions, where bioclasts remain over long periods in the taphonomic active zone. In this context, background processes were mostly destructive due to: 1) physical destruction as a consequence of the high hydraulic energy, 2) biological destruction due to intense boring, and 3) chemical destruction due to early dissolution of the unstable calcium carbonate phases. These taphonomic processes are commonly affecting fossil preservation in temperate carbonate platforms. The Q-mode cluster analysis separates a group of samples characterized by high taphonomic destruction as a consequence of the aforementioned environmental processes. In addition to the background normal processes, event processes, such as storms, produced sedimentological (tempestite beds) and taphonomic signatures. Two samples, corresponding to tempestite beds, are separated in the Q-mode cluster analysis. Finally, the cluster separates a third group of samples characterized by the abundance of moulds. An early cementation played a key role in the formation of this assemblage.
\end{abstract}

Keywords: Taphonomy, taphofacies, temperate carbonates, Pliocene, Almayate, Málaga.

\section{RESUMEN}

Se analizan los procesos tafonómicos que afectan a las asociaciones de fósiles de los carbonatos templados del Plioceno que afloran en la Cuenca de Almayate (Málaga, S de España). El estudio tafonómico se ha realizado cuantificando varias propiedades tafonómicas. Los valores obtenidos han servido para realizar un análisis multivariante de agrupamiento de muestras, cluster en modo Q, con objeto de establecer modelos de tafofacies. Los resultados obtenidos indican unas condiciones de depósito de alta energía donde los restos esqueléticos permanecieron durante prolongados períodos de tiempo en la zona tafonómicamente activa. En este contexto, durante los periodos de sedimentación normal (background processes) se produjo un efecto combinado de destrucción tafonómica: 1) destrucción física, como consecuencia de la alta energía, 2) destrucción biológica, debido al efecto de los organismos bioperforadores, y 3) destrucción química, por disolución de las fases inestables del carbonato cálcico. Estos procesos tafonómicos son los que de forma habitual afectan a los carbonatos templados. En el análisis cluster realizado se separa un grupo de muestras caracterizadas por una alta destrucción tafonómica debido a la interacción de estos procesos. Además de estos procesos normales, la acción esporádica de tormentas (event processes) dejó señales sedimentológicas (presencia de niveles de tempestitas) y tafonómicas propias. En este sentido, el análisis cluster individualiza un conjunto de muestras tomadas en niveles de tempestitas. Finalmente, el análisis cluster separa un tercer grupo de muestras donde domina la presencia de moldes de organismos originalmente aragoníticos sobre restos que mantienen la mineralogía original de la concha. Una cementación temprana debió jugar un papel decisivo en la formación de esta asociación.

Palabras claves: Tafonomía, tafofacies, carbonatos templados, Plioceno, Almayate, Málaga. 


\section{INTRODUCCIÓN}

Los sedimentos carbonatados marinos ocupan aproximadamente el 50\% de los fondos oceánicos actuales (Tucker \& Wright, 1992). Durante la década de los sesenta y buena parte de la década de los setenta, la inmensa mayoría de los estudios se centraron en los carbonatos someros tropicales (Ginsburg, 1956; Purdy, 1963; Shinn et al., 1969; Davies, 1970), lo que llevó a la idea errónea de que estos materiales estaban restringidos a latitudes bajas. Esta visión cambió con los trabajos pioneros de Chave (1967) y Lees \& Buller (1972), quienes demostraron que pueden encontrarse carbonatos marinos en cualquier latitud. Lees \& Buller (1972) proponían que los carbonatos de ambientes tropicales y de aguas más frías se pueden diferenciar por los componentes bióticos que contribuyen mayoritariamente a su producción; asociaciones "chlorozoan" y "foramol" respectivamente. Esta división simple ha sido refinada y ampliada posteriormente (Nelson et al., 1988a; Carannante et al., 1988; Hayton et al., 1995). James (1997) propone los términos "heterozoan" y "photozoan" para los carbonatos de zonas templadas y tropicales respectivamente.

Un factor clave en la formación de carbonatos tropicales o aquellos caracterizados por asociaciones de heterozoos es la temperatura (Lees \& Buller, 1972; Nelson, 1988; James \& Clarke, 1997; Sánchez-Almazo et al., 2001; Pedley \& Carannante, 2006; Martín et al., 2009a). No obstante, hay determinados factores que pueden llegar a modificar los efectos del control climático como son la profundidad (Carannante et al., 1988; Pomar et al., 2004), el contenido en nutrientes (Hallock, 1981; Mutti et al., 1997; Mutti \& Hallock, 2003) o la existencia de corrientes de aguas frías (Martín et al., 2009a, 2009b).

Una particularidad de las plataformas carbonatadas templadas con relación a las tropicales es que en las primeras la tasa de sedimentación es muy baja y, además, la precipitación inorgánica y la cementación marina es inexistente o muy reducida (Smith \& Nelson, 2003). Estas características hacen que los ambientes templados sean esencialmente destructivos debido a un prolongado tiempo de residencia de los restos esqueléticos en la zona tafonómicamente activa y a que dominan los procesos de disolución de las conchas con mineralogías inestables (aragonito y calcita con alto contenido en $\mathrm{Mg}$ ). Como consecuencia, las asociaciones de fósiles en los carbonatos templados muestran marcados sesgos de conservación (Smith \& Nelson, 2003). Estos autores hacen un amplio análisis y una exhaustiva revisión de los procesos tafonómicos que afectan a las asociaciones de organismos en plataformas carbonatadas templadas actuales.

En las cuencas neógenas de la Cordillera Bética se encuentra un buen registro sedimentario de carbonatos templados (ver resúmenes recientes en Braga et al., 2006, y Martín et al., 2009a). Si bien la sedimentología de estos depósitos y su distribución secuencial durante el Neógeno se conocen con gran detalle (Martín et al., 1996, 2004, 2009a, 2009b; Betzler et al., 1997, 2000; Aguirre, 2000; Braga et al., 2001, 2003, 2006, 2010; Puga-Bernabéu et al., 2007, 2008, 2010), los estudios de tafonomía son más escasos (Aguirre, 1992, 1996; Aguirre \& Jiménez, 1997, 1998; Aguirre \& Yesares-García, 2003; Yesares-García \& Aguirre, 2002, 2004; Rico et al., 2008; Aguirre et al., 2008).

En la Cuenca de Almayate (Málaga, S de España), afloran carbonatos templados con alguna influencia de aportes puntuales de material terrígeno de edad Plioceno inferior (Mayoral \& Rodríguez-Vidal, 1994; Aguirre, 2000). Aguirre (2000) hizo una primera valoración cualitativa de los atributos tafonómicos observados en estos materiales para inferir las condiciones de depósito en las que se formaron y proponer un modelo de evolución secuencial. En este trabajo, hacemos un estudio cuantitativo de las propiedades tafonómicas con objeto, no sólo de reconstruir las condiciones paleoambientales, sino también de establecer modelos de tafofacies. Los modelos de tafofacies resultantes servirán para comparar con los ya establecidos por otros autores en otras cuencas y contextos paleoambientales. Así mismo, se discute cómo el estudio cuantitativo realizado permite identificar condiciones de depósito particulares, donde parece que los mecanismos de conservación fueron más importantes que los procesos destructivos en un contexto de carbonatos templados.

\section{LOCALIZACIÓN GEOGRÁFICA Y CONTEXTO GEOLÓGICO}

La sección estudiada se localiza en la Atalaya, entre las localidades de Almayate y Torre del Mar, en la costa de Málaga (Fig. 1). Se trata de un excelente afloramiento donde los materiales pliocenos se exponen en continuidad lateral a lo largo de varios kilómetros.

El basamento de la Cuenca de Almayate está constituido por rocas metamórficas del Complejo Maláguide. El relleno sedimentario, materiales objeto de estudio, lo forman rocas carbonatadas y siliciclásticas de edad Plioceno inferior (Fig. 1). Estos sedimentos rellenan un relieve fuertemente excavado en el basamento mediante un contacto de solapamiento expansivo. La naturaleza carbonatada de los materiales estudiados es una singularidad en relación con el resto de cuencas pliocenas formadas a lo largo de la costa malagueña, pequeñas cuencas emergidas pertenecientes al margen septentrional del Mar de Alborán. En general, el Plioceno malagueño está constituido por depósitos terrígenos, limos, arenas y conglomerados y sólo localmente, arcillas (por ejemplo, Serrano, 1993; Aguirre et al., 2005). Tan sólo en las pequeñas cuencas de Almayate y alrededores (antigua Estación de Valle-Niza) y en la Cuenca de Nerja (playa Burriana) se observan materiales carbonatados o mixtos carbonatados-siliciclásticos 


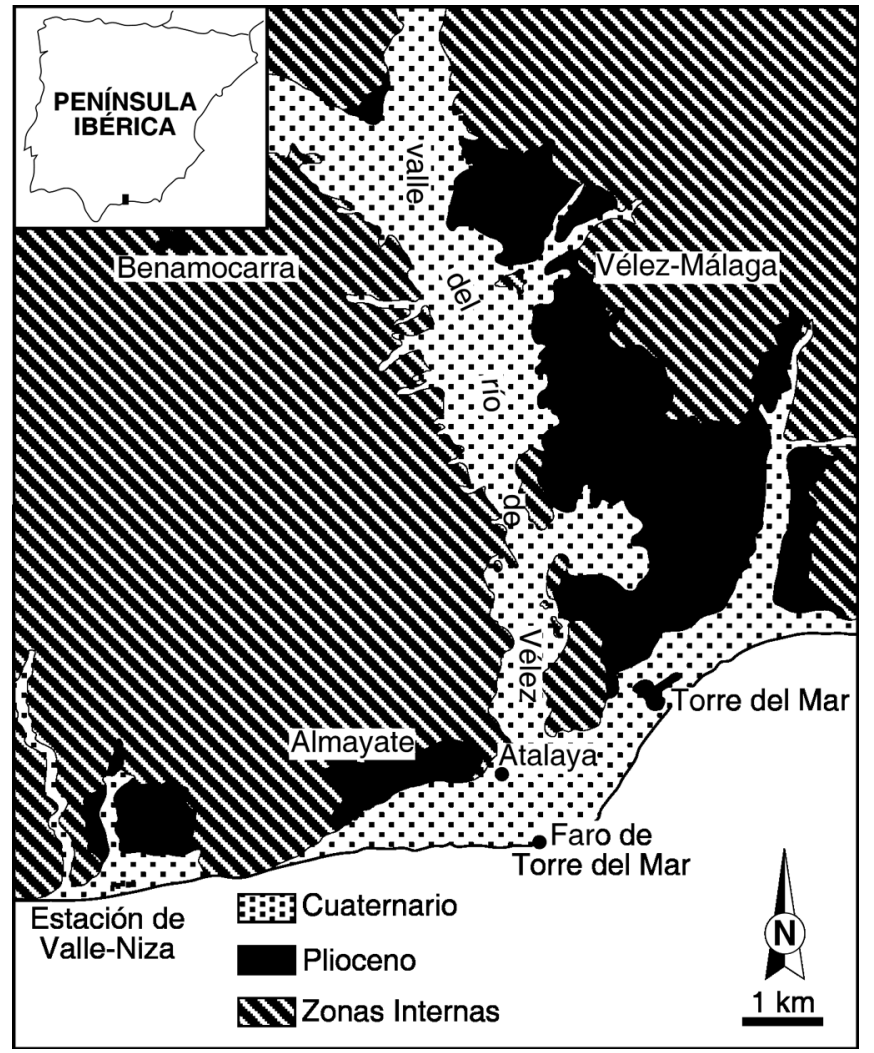

Figura 1. Mapa geológico de la Cuenca de Almayate con la localización de la sección estudiada, La Atalaya (tomado de Aguirre, 2000).

Geological map of the Almayate Basin with indication of the studied section, La Atalaya (from Aguirre, 2000).

(Mayoral \& Rodríguez-Vidal, 1994; Aguirre, 2000). Esta particularidad se debe a la configuración paleogeográfica de la zona (Guerra-Merchán \& Serrano, 1993; Aguirre, 2000). Dichas áreas quedaron aisladas de la influencia de grandes sistemas terrígenos (deltas y abanicos deltaicos) que se distribuyeron a lo largo de la costa y que alimentaban con material siliciclástico todo el margen norte de Alborán durante el Plioceno. Únicamente, pequeños abanicos deltaicos descargaron localmente material terrígeno que se mezcló con la sedimentación carbonatada. Los carbonatos de estas cuencas están dominados por abundantes restos de bivalvos y briozoos y se pueden atribuir a la litofacies de carbonatos templados "molechfor" de Carannante et al. (1988).

Los depósitos pliocenos en la sección de la Atalaya alcanzan unos $60 \mathrm{~m}$ de espesor (Fig. 2). En este punto, extremo oriental de la cuenca, el Plioceno se dispone con un buzamiento entre $25^{\circ}$ y $30^{\circ}$ al $\mathrm{O}$, que disminuye a menos de $10^{\circ}$ hacia el centro de la cuenca, en las proximidades de Almayate (Fig. 3). En el extremo occidental de la cuenca, el buzamiento es de aproximadamente $30^{\circ}$ hacia el E, que disminuye en ese sentido. Este dispositivo le confie-

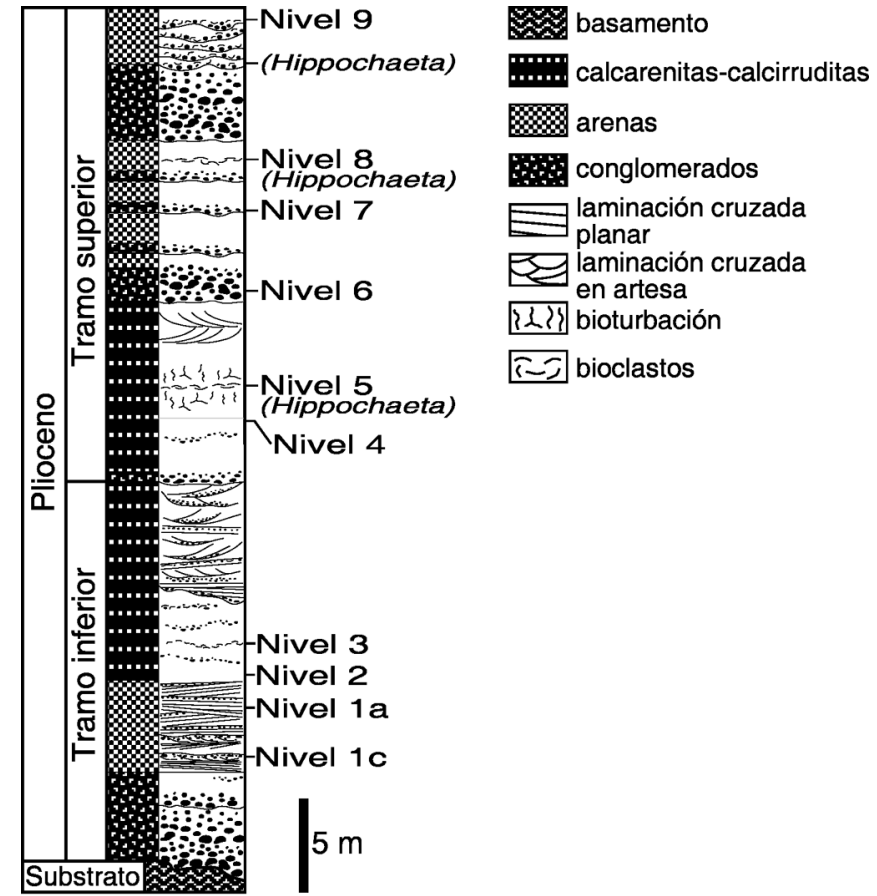

Figura 2. Columna estratigráfica de la sección La Atalaya indicando los niveles seleccionados para hacer las observaciones tafonómicas (modificada de Aguirre, 2000). Stratigraphic column of the La Atalaya section indicating the different beds (nivel) selected for the taphonomic study (modified from Aguirre, 2000).

re al conjunto de materiales una geometría cóncava hacia arriba. El estudio detallado de la estratigrafía, la sedimentología, el análisis de facies y la evolución secuencial del Plioceno de la Cuenca de Almayate ha sido realizado por Aguirre (2000). Es por ello que a continuación se ofrece una descripción de las características generales del Plioceno, centrándonos en las propiedades tafonómicas de los niveles estudiados.

\section{METODOLOGÍA}

El estudio tafonómico llevado a cabo sigue una aproximación metodológica combinada usando datos cualitativos, semicuantitativos y cuantitativos según el protocolo de muestreo propuesto por Yesares-García \& Aguirre (2004). En primer lugar se procedió a determinar los niveles fosilíferos en los que realizar los análisis semicuantitativos y cuantitativos. Se hizo una descripción de los mismos en función de la composición taxonómica, la fábrica, la geometría y la estructura interna según la terminología propuesta por Kidwell et al. (1986). Posteriormente, una vez establecidos los diferentes niveles de muestreo, se estimaron los valores de empaquetamiento bioclástico y del porcentaje de fósiles por volumen de roca usando las tablas creadas para tales efectos por Kidwell \& Holland (1991). 


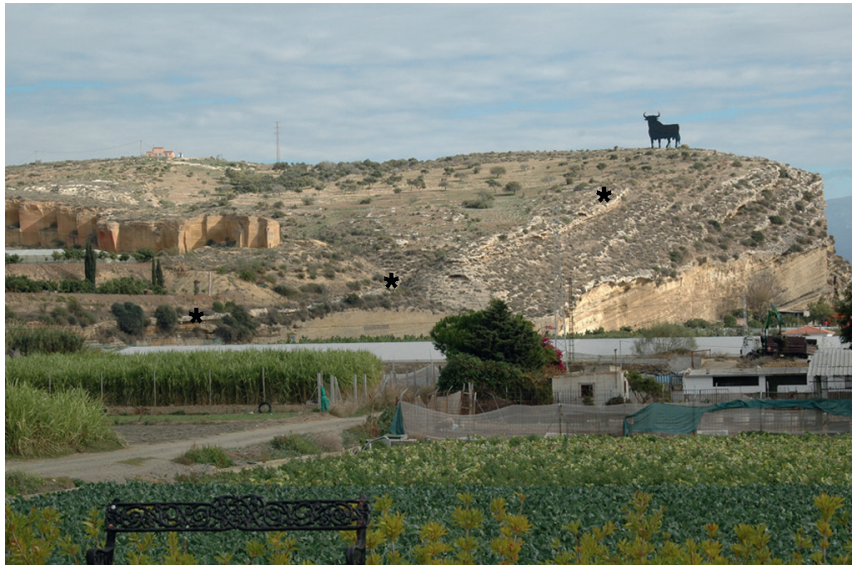

Figura 3. Vista panorámica (mirando hacia el E) del afloramiento estudiado. Las capas se disponen horizontalmente hacia el centro de la cuenca, como se puede ver en la capa marcada con asteriscos.

Panoramic view (looking to the east) of the studied outcrop. The dipping of the strata decreases towards the centre of the basin, as observed in the bed marked with asterisks.

Finalmente, se cuantificaron diferentes atributos tafonómicos como articulación, fragmentación, orientación preferencial (midiendo el ángulo que forman los restos bioclásticos con la estratificación, la orientación de la concavidad y la existencia de restos orientados en su posición original de crecimiento), pulido o desgaste de los bordes de los fragmentos, pérdida de la ornamentación y otros debidos a interacciones biológicas (presencia de bioperforaciones o encostrantes). Además, se estimó el número de fósiles como una indicación de la abundancia y riqueza, y el tamaño de los restos, indicativo del nivel de clasificación por tamaños (granoselección).

Las medidas de los atributos tafonómicos se realizaron en diferentes estaciones de muestreo en niveles distribui- dos a lo largo de la sección estudiada (Fig. 2). El criterio para seleccionar las diferentes estaciones de muestreo fue incluir en el estudio tafonómico todas las facies y litologías posibles. Se seleccionaron diferentes tipos de facies: a) facies de conglomerados y arenas medias-gruesas con estratificación cruzada (Niveles 1a, 1c; Fig. 4a); b) facies de calcarenitas-calcirruditas con estratificación cruzada (Niveles 2 y 4); c) facies de conglomerados fosilíferos canalizados (Niveles 6 y 7; Fig. 4b); d) facies no canalizadas de acumulación de fósiles (Niveles 5 y 8; Figs. 4c4d); y e) facies canalizadas con concentraciones basales residuales (lag) de conchas (Niveles 3 y 9; Figs. 4e-4g). En cada estación de muestreo se midieron las propiedades tafonómicas en 10 cuadrados de $20 \mathrm{~cm}$ de lado distribuidos al azar y según la estratificación. Se contabilizaron todos los restos mayores de $0,5 \mathrm{~cm}$. Dicho tamaño se seleccionó arbitrariamente ya que es el tamaño mínimo para identificar de forma óptima los fósiles al menos a nivel taxonómico superior.

Los valores promedios de las medidas en los 10 cuadrados obtenidos en cada estación de muestreo se presentan en la Tabla 1. Mediante un análisis de estadística multivariante de agrupamiento de muestras, análisis cluster en modo Q, se obtuvo una aproximación al análisis de tafofacies ya que en el dendrograma se agrupan las diferentes estaciones de muestreo en función de las propiedades tafonómicas. El análisis cluster se realizó usando el método de la media (average) y distancias euclideas (Euclidean distances) (Meldahl \& Flessa, 1990; Yesares-García \& Aguirre, 2004).

\section{TAFONOMÍA}

\section{RESULTADOS CUANTITATIVOS}

La sección de la Atalaya puede dividirse en dos tramos (Fig. 2). El tramo inferior, de unos $30 \mathrm{~m}$ de espesor máximo, comienza con unas brechas y conglomerados

Figure 4. Fotos de campo de algunas facies o aspectos tafonómicos característicos. a: Facies de arenas con estratificaciones cruzadas; el martillo marca una concentración residual de ostras (restos blanquecinos). b: Canal de conglomerado fosilífero dominado por pectínidos. c: Nivel de concentración de Hippochaeta (nivel 5); el hueco que se observa hacia la derecha del nivel corresponde al molde de un individuo completo y articulado. d: Detalle de un individuo articulado de Hippochaeta que conserva parte de la concha original. e: Concentración de pectínidos con base canalizada interpretada como tempestitas; el contenido en terrígenos y su posición estratigráfica indica que se trata de una tempestita proximal. Obsérvese la disposición empaquetada (stacking) de los pectínidos con la concavidad preferentemente orientada hacia el techo de la capa. f: Diversos niveles de tempestitas distales formadas por concentraciones de pectínidos. g: Detalle de una tempestita distal donde se puede observar la disposición empaquetada de las conchas (stacking) y la orientación preferente de la concavidad hacia el techo. h: Intensa bioturbación, dominando el icnogénero Thalassinoides, asociada al nivel 5, concentración de Hippochaeta. Field views of some facies and taphonomic attributes. a: Sands with cross bedding facies; the hammer points to an oysters lag-deposit (the whitish shells). $\boldsymbol{b}$ : Channel of fossiliferous conglomerate containing abundant pectinids. c: Hippochaeta shell concentration (bed 5); the void at the right margin of the bed is the cast of a complete and articulated individual. $\boldsymbol{d}$ : Close-up view of an articulated Hippochaeta with the original shell preserved. $\boldsymbol{e}$ : Lag of pectinids interpreted as a storm bed; the terrigenous content and the stratigraphic position of the bed indicate that it is a proximal tempestite. Note the dominant stacked biofabric and the preferred concave-up orientation of the shells. $f$ : Distal tempestites formed by pectinid concentrations. $g$ : Close-up view of one of the distal tempestites; note the stacked biofabric and the preferred concave-up orientation of the shells. $\boldsymbol{h}$ : Bioturbation, ichnogenus Thalassinoides, associated with bed 5, the Hippochaeta concentration. 

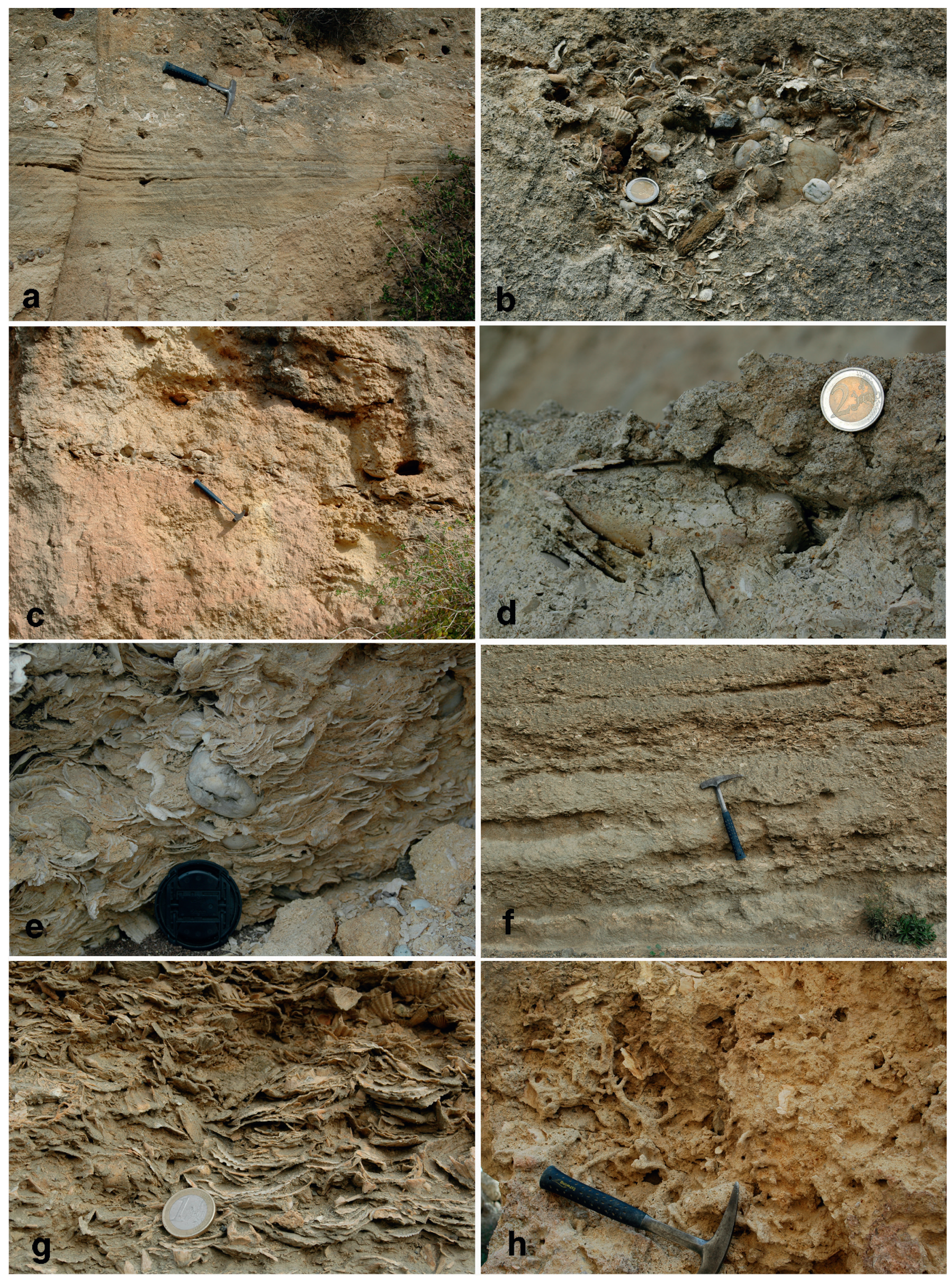
Tabla 1. Atributos tafonómicos medidos en la sección de La Atalaya. Todos los valores se expresan en porcentajes. frag. $=$ fragmentación; complet. = completos; art. = articulados; vert. $=$ restos orientados verticalmente; plano $=$ restos sin una concavidad determinable; perfor. = perforaciones; encos. = encostrantes.

Taphonomic signatures measured in the La Atalaya section. All values are in percentages. frag. = fragmentation; complet. = complete; art. = articulation; vert. = bioclasts vertically orientated; plano = bioclasts with unidentifiable concavity; perfor. = borings; encros. = encrustings.

\begin{tabular}{|c|c|c|c|c|c|c|c|c|c|c|c|c|c|c|c|}
\hline \multirow{2}{*}{ estación } & \multirow{2}{*}{\begin{tabular}{|c|}
$\mathrm{n}^{\mathrm{o}}$ \\
fósiles
\end{tabular}} & \multirow{2}{*}{ frag. } & \multirow{2}{*}{ complet. } & \multirow{2}{*}{ art. } & \multicolumn{4}{|c|}{ orientación concavidad } & \multicolumn{2}{|c|}{ conservación concha } & \multicolumn{3}{|c|}{ aristas } & \multicolumn{2}{|c|}{ interacciones } \\
\hline & & & & & -1 & - & vert. & plano & original & molde & aguda & redondeada & sin aristas & perfor. & encos. \\
\hline nivel 1a & 109 & 97,1 & 2,9 & 1,9 & 32,4 & 11,4 & 24,8 & 29,5 & 100 & 0 & 0 & 99 & 1 & 36,4 & \\
\hline nivel 1c & 97 & 99 & 1 & 1 & 17,7 & 30,2 & 29,2 & 21,9 & 100 & 0 & 0 & 100 & 0 & 45,4 & 6,2 \\
\hline nivel 2 & 106 & 100 & 0 & 0 & 21,6 & 18,6 & 25,5 & 34,3 & 100 & 0 & 0 & 100 & 0 & 16 & \\
\hline nivel 3 & 233 & 100 & 0 & 0 & 24,9 & 24,9 & 23,1 & 27,1 & 100 & 0 & 0 & 100 & 0 & 3,4 & \\
\hline nivel 4 & 188 & 100 & 0 & 0 & 10,8 & 13,1 & 27,8 & 48,3 & 9,1 & 90,9 & 0,6 & 8 & 91,5 & 1,1 & \\
\hline nivel 5 & 285 & 98,6 & 1,4 & 1,1 & 15,9 & 16,6 & 25,3 & 41,2 & 7,9 & 92,1 & 0,4 & 7,6 & 92,1 & & \\
\hline nivel 6 & 104 & 100 & 0 & 0 & 29,1 & 9,7 & 53,4 & 7,8 & 100 & 0 & 0 & 100 & 0 & & \\
\hline nivel 7 & 168 & 98,6 & 1,4 & 0 & 25,3 & 12,3 & 25,3 & 37 & 100 & 0 & 0 & 100 & 0 & & \\
\hline nivel 8 & 175 & 99,4 & 0,6 & 0,6 & 24 & 28 & 18,3 & 29,1 & 10,9 & 88,6 & 0 & 10,9 & 88,6 & 6,3 & \\
\hline nivel 9 & 250 & 100 & 0 & 0 & 47 & 22,9 & 20,9 & 9,2 & 100 & 0 & 0 & 100 & 0 & 0,8 & 0,4 \\
\hline
\end{tabular}

rojos que descansan directamente sobre el basamento paleozoico, adaptándose y enrasando el paleorrelieve, y se acuñan hacia el S y el ENE. No se han encontrado fósiles.

Hacia arriba se pasa gradualmente a unas brechas y conglomerados amarillentos, con superficies erosivas internas y granoselección positiva. Según se sube en la sección se intercalan paquetes de arenas medias-gruesas entre los conglomerados que presentan estratificación cruzada tabular de bajo ángulo, con un sentido de paleocorriente hacia el S. Tanto los conglomerados como las arenas contienen mayoritariamente Ostrea edulis (Linné, 1758) (Fig. 4a). En los conglomerados (nivel 1c; Fig. 2) la fauna aparece dispersa, representando un 5\% del volumen de roca. La totalidad de los restos conserva concha calcítica (Tabla 1). Tan sólo el 1\% del total de la fauna está completa y, además, articulada. Hay una fuerte tendencia a la verticalidad (Fig. 5), y ausencia de selección de tamaños (Fig. 5). Predominan los restos dispuestos con la concavidad hacia abajo. Todos los fragmentos presentan las aristas redondeadas. El $45,4 \%$ está afectado por endobiontes y el $6,2 \%$ por encostrantes.
En las arenas con estratificaciones cruzadas (nivel 1a; Fig. 2) se observa el mismo empaquetamiento (disperso) y porcentaje de restos por volumen de roca $(5 \%)$ que en los conglomerados. El 100\% de los restos presenta concha calcítica (Tabla 1). El porcentaje de restos completos es del 2,9\% y el de articulación algo más bajo, el 1,9\% del total de la asociación. Se observa una orientación bipolar, con un máximo de restos orientados perpendicularmente, $49,5 \%$, y oblicuamente, $36,2 \%$ (Fig. 5). No hay ninguna selección de tamaños (Fig. 5). Los restos orientados con la concavidad hacia arriba son los más numerosos. Todos los fragmentos tienen las aristas redondeadas. El 36,4\% del total de la asociación muestra marcas de endobiontes.

El contenido en terrígenos disminuye progresivamente hacia techo y se pasa a calcirruditas bioclásticas muy cementadas y con estratificación cruzada en artesa con un alto contenido en siliciclásticos. Los restos mayores de $0,5 \mathrm{~cm}$ son escasos y se encuentran dispersos en la matriz, con un porcentaje inferior al $10 \%$ del volumen. Tan sólo hay fragmentos con concha calcítica y con las aristas redondeadas (nivel 2; Tabla 1). Están dispuestos con una

Figura 5. Histogramas de frecuencia de las propiedades tafonómicas analizadas. En la columna de histogramas de la izquierda se indica el ángulo de inclinación de los restos con relación a la superficie de estratificación. En la siguiente columna de histogramas se indican los porcentajes promedio de diferentes propiedades tafonómica (vol. = porcentaje de bioclastos por volumen de roca; frag. = fragmentación; art. = articulados; mold. = moldes; abr. = desgaste). La siguiente columna muestra la orientación de la concavidad $(v=$ concavidad hacia arriba; $n=$ concavidad hacia abajo; $\mid=$ restos verticales; $\mathrm{P}=$ restos planos). La última columna de histogramas indica la selección de tamaños de bioclastos.

Frequency histograms of the measured taphonomic attributes. In the left column of histograms, it is indicated the angle formed by the fossil remains with the stratification. The next column of histograms shows the average percentage of several taphonomic attributes (vol. = percentage of remains by rock volume; frag. = fragmentation; art. = articulation; mold. $=$ moulds; abr. = abrasion). Next column of histograms indicates the concavity orientation ( $=$ concave up; $\cap=$ concave down; $\mid$ = vertically oriented remains; $P$ = remains with no defined concavity). The last column of histograms shows bioclast sorting. 
Nivel 1a
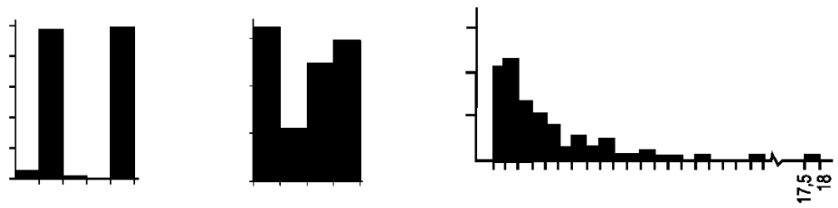

Nivel 1c
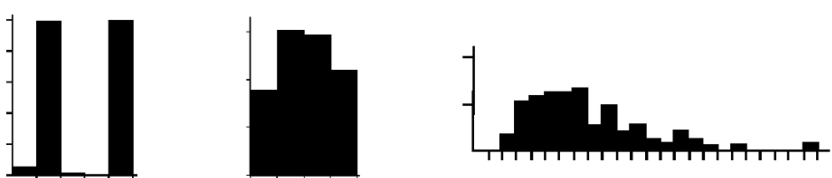

Nivel 2
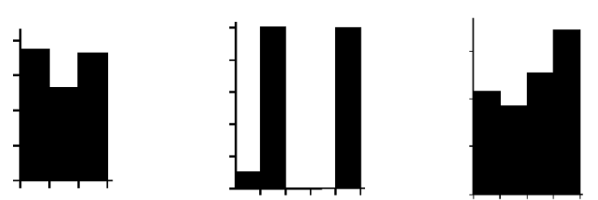

Nivel 3
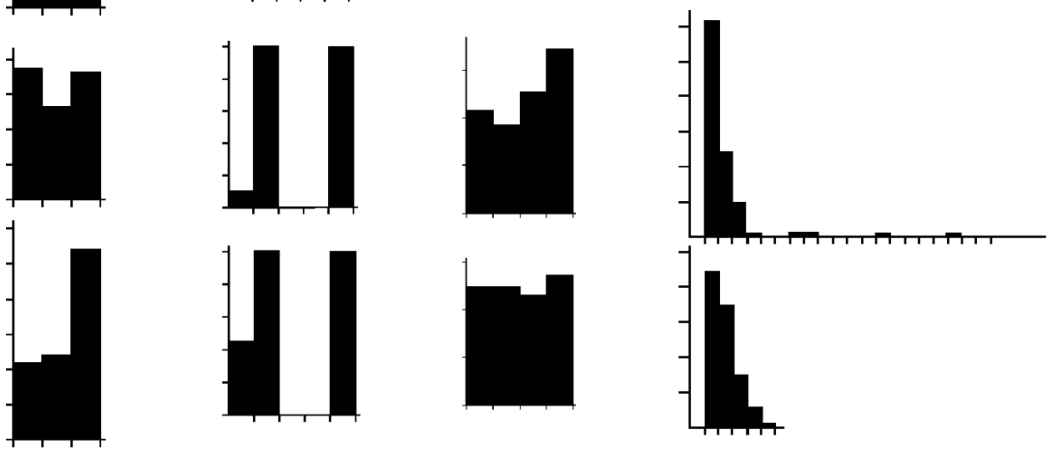

Nivel 4
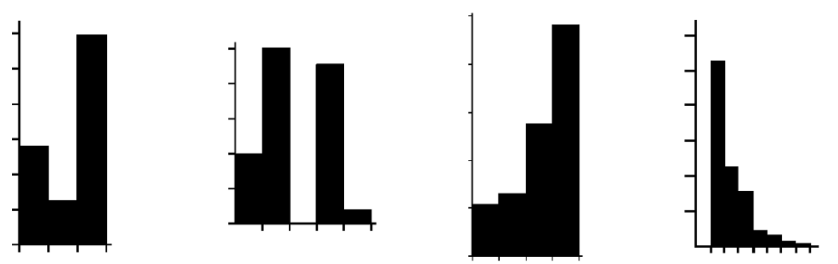

Nivel 5
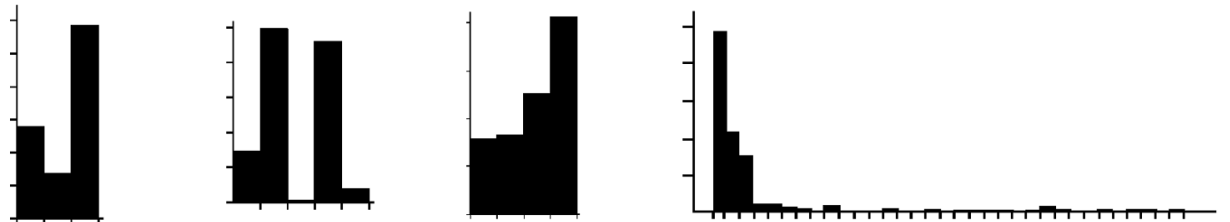

Nivel 6
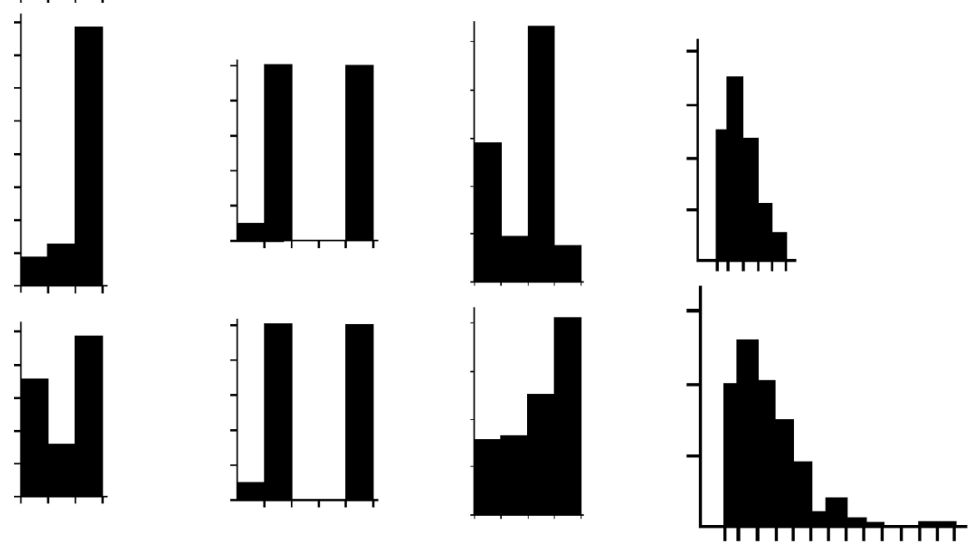

Nivel 8
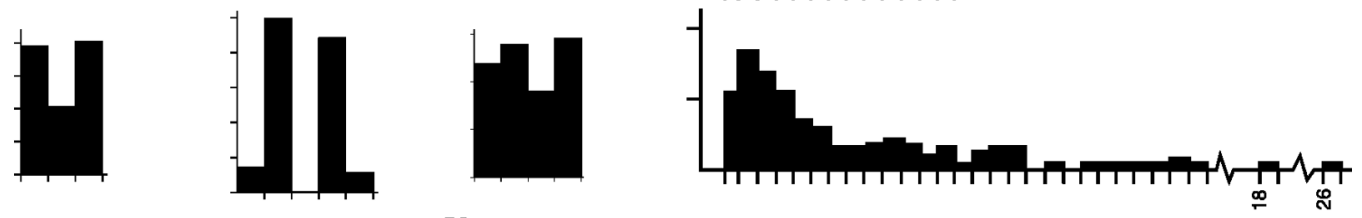

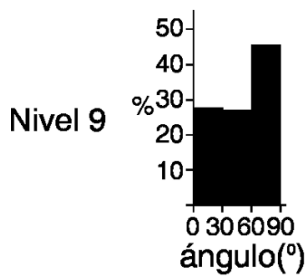
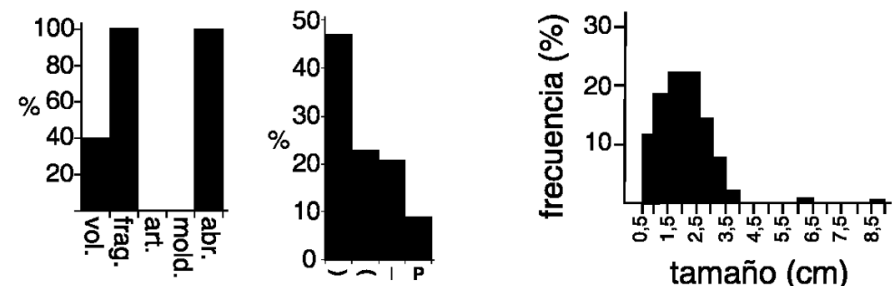
orientación bipolar, dominando restos orientados vertical y concordantemente (Fig. 5). Hay un claro dominio de restos planos; entre los que tienen una curvatura definida, hay una mayor proporción de restos orientados con la concavidad hacia arriba. Hay una buena selección de tamaños, con un máximo de restos entre 0,5 y $1,5 \mathrm{~cm}$ de diámetro (Fig. 5). No se ha detectado ningún encostramiento y el $16 \%$ está afectado por bioperforaciones.

Entre las calcirruditas se intercalan capas poco potentes (hasta $10 \mathrm{~cm}$ de espesor máximo) con bases erosivas, gradación normal y acumulaciones residuales de fósiles (nivel 3). Tienen un empaquetamiento denso, con el $45 \%$ del volumen. Al igual que en la matriz circundante, sólo aparecen fragmentos de fósiles calcíticos con las aristas redondeadas y textura pulverulenta debido a la disolución (nivel 3; Tabla 1). Hay una clara orientación preferencial vertical (Fig. 5) y una selección de tamaños de bioclastos relativamente buena (Fig. 5). Como en el nivel anterior, dominan los restos que no tienen una concavidad definida. Entre los restos curvos no hay una orientación preferencial de la concavidad. En el total de la asociación predominan los restos planos. Tan sólo un 3,4\% del total de la fauna presenta trazas de bioperforaciones.

El segundo tramo, de $30 \mathrm{~m}$ de espesor, comienza con un paquete de conglomerados de grandes bloques (hasta $20 \mathrm{~cm}$ de diámetro máximo) y con gradación normal dispuesto sobre una superficie erosiva. Por encima se pasa de forma rápida, aunque gradual, a calcirruditas detríticas. Se intercalan algunos conglomerados fosilíferos con bases canalizadas y granoselección normal. Esta primera parte del tramo aflora en paredes verticales de antiguas canteras, por lo que es imposible acceder a ellas.

La cantidad de siliciclásticos disminuye hacia techo y, de forma concomitante, aumenta el contenido en fósiles pasándose a unas calcarenitas muy fosilíferas y con estratificación cruzada en artesa. Predominan diversas especies del género Chlamys Röding, 1798. Muestran un empaquetamiento denso, con un porcentaje de restos del $40 \%$ del volumen. Dominan los restos conservados como moldes, 90,9\% (nivel 4; Tabla 1). Los restos calcíticos tienen un aspecto pulverulento, conservándose sólo algunas capas de la concha. La totalidad de la asociación está constituida por fragmentos, de los cuales sólo el $0,6 \%$ de los restos calcíticos tiene las aristas agudas. Hay una clara orientación preferencial perpendicular (Fig. 5) y una buena selección de tamaños (Fig. 5). Los restos planos son los más abundantes en el total de la asociación, 48,3\%. Entre los restos con una curvatura definida, abundan ligeramente los restos dispuestos con la concavidad hacia abajo. El 1,1\% está afectado por bioperforaciones.

Dentro del tercio inferior de este segundo tramo, se encuentra un nivel de concentración de grandes individuos de Hippochaeta sp. incluidos en una matriz calcarenítica masiva y bioturbada (Figs. 4c-4d y 4h). Este nivel (nivel 5; Fig. 2), de $15 \mathrm{~cm}$ de espesor, tiene una geometría tabular. Junto a Hippochaeta Sangiovanni, 1884 también hay abun- dantes fragmentos de Chlamys spp. con la concha parcialmente disuelta. Presenta un empaquetamiento denso, con el $30 \%$ de fósiles por volumen de roca. Las propiedades tafonómicas no varían sustancialmente de aquellas medidas en la matriz calcarenítica en la que se incluye (Tabla 1). Tan sólo es de resaltar la gran dispersión de tamaños de bioclastos hacia las clases de tamaños mayores debido a la talla que alcanzan los ejemplares de Hippochaeta (Fig. 5). También difieren en la presencia de algunos individuos completos $(1,4 \%)$ y otros articulados $(1,1 \%)$ en el total de la asociación (Fig. 4d).

Por encima del nivel de Hippochaeta se pasa nuevamente a unas calcarenitas bioclásticas con estratificaciones cruzadas en artesa y aumenta progresivamente el contenido en material terrígeno (Fig. 2). Además de este aumento en el contenido en material siliciclástico en la matriz calcarenítica, se intercalan paquetes de conglomerados con bases erosivas y gradación normal. Cada banco está constituido por acumulaciones residuales de conglomerados fosilíferos que cambian hacia arriba a arenas medias-gruesas sin apenas fósiles. Así mismo, se intercalan niveles con bases canalizadas donde se forman concentraciones residuales de fósiles. Cabe destacar la presencia de dos niveles de concentración de Hippochaeta (Fig. 2) y otros niveles dominados casi exclusivamente por diferentes especies de pectínidos, dominando los géneros Aequipecten Fisher, 1886 y Chlamys.

En los paquetes de conglomerados fosilíferos hay un dominio de ostreidos y pectínidos, con un empaquetamiento disperso a poco denso, entre el $5 \%$ y el $10 \%$ del volumen. Sólo se encuentran fragmentos calcíticos con las aristas redondeadas (niveles 6 y 7; Tabla 1). En el nivel 6 dominan los restos con una orientación preferencial perpendicular $(78,6 \%)$ mientras que en el nivel 7 hay una orientación bipolar (Fig. 5). Se observa una buena selección de tamaños de bioclastos (Fig. 5). Dominan los fragmentos orientados con la concavidad hacia arriba. Tan sólo algunos fósiles están afectados por bioperforaciones $\mathrm{y}$ encostrantes.

Una estación de muestreo se localizó en otro de los niveles de Hippochaeta de la parte alta de la sección (nivel 8; Fig. 2). Se caracteriza por un empaquetamiento poco denso y un $15 \%$ del volumen. Dominan los moldes, $89,1 \%$ (Tabla 1). Sólo el 0,6\% del total está completo y articulado. Hay una marcada bipolaridad en la orientación de los fósiles (Fig. 5) y una gran dispersión de tamaños (Fig. 5). Si bien son más abundantes los restos planos, entre los que tienen curvatura hay una proporción muy similar de restos orientados con la concavidad hacia arriba y hacia abajo. Todos los fragmentos conservados con concha presentan las aristas redondeadas (10,9\%). El 6,3\% del total está afectado por endobiontes.

En las acumulaciones densas de pectínidos del techo de la sección el porcentaje de restos oscila entre el $35 \%$ y el $40 \%$ del volumen de roca (nivel 9; Tabla 1). Es bastante 
frecuente encontrar concentraciones de valvas desarticuladas dispuestas unas sobre otras a modo de recipientes encajados (Fig. 4e), es decir, con un ordenamiento stacking según Kidwell et al. (1986). La totalidad de los restos son fragmentos con aristas redondeadas de organismos de conchas calcíticas. No obstante, muestran un cierto grado de disolución ya que las conchas tienen un aspecto pulverulento y sólo se conservan algunas capas internas de la concha original. Hay una orientación preferencial perpendicular, con un $45,4 \%$ de restos orientados con un ángulo superior a $60^{\circ}$ (Fig. 5). La selección de tamaños es relativamente buena (Fig. 5). Los fragmentos orientados con la concavidad hacia arriba son los más abundantes (Figs. 4e y 4g). Los porcentajes de restos con bioperforaciones o encostrados son notablemente bajos, $0,8 \%$ y $0,4 \%$ respectivamente.

\section{ANÁLISIS MULTIVARIANTE}

El análisis cluster en modo Q realizado separa tres agrupaciones bien diferenciadas. El cluster 1 aúna las muestras de los niveles 1a, 1c, 2, 6 y 7 (Fig. 6). Desde un punto de vista de facies, esta agrupación se caracteriza por unir estaciones de muestreo localizadas en arenas gruesas y conglomerados fosilíferos. Las propiedades tafonómicas que caracterizan este cluster son una baja proporción de fósiles por volumen de roca $(5-10 \%)$, alta dispersión de restos, ausencia de moldes y de granoclasificación, restos dispuestos verticalmente y sin una orientación preferencial con relación a la concavidad y una alta tasa de bioperforaciones y encostrantes (Fig. 5).

El cluster 2 incluye las muestras de los niveles 3 y 9 (Fig. 6). Se trata de niveles canalizados de concentración de fósiles, donde los restos se encuentran formando lags en la base de las zonas canalizadas. Las propiedades tafonómicas características de este cluster son concentraciones densas, con hasta el $40 \%$ de restos por volumen de roca, ausencia de moldes, buena granoselección de bioclastos y bajos porcentajes de colonizaciones (Fig. 5).

Finalmente, el cluster 3 agrupa los niveles 4,5 y 8 (Fig. 6). En concreto, los niveles 5 y 8 se corresponden con niveles de concentración de Hippochaeta y el nivel 4 se localiza en los depósitos de calcarenitas-calcirruditas inmediatamente por debajo del nivel 5 (Fig. 2). Los atributos tafonómicos característicos de este cluster son concentraciones densas, con hasta el $40 \%$ de restos por volumen de roca, predominio de moldes, mala granoselección de bioclastos y bajos porcentajes de colonizaciones (Fig. 5).

\section{DISCUSIÓN}

\section{INTERPRETACIÓN DE CONJUNTO}

El estudio tafonómico de los materiales pliocenos de Almayate pone de manifiesto la alta fragmentación, abrasión y desarticulación que han sufrido los fósiles. En general, estas características tafonómicas indican un efecto combinado de una larga exposición de los restos en la zona tafonómicamente activa en un medio de depósito marino somero sometido a una alta energía hidráulica. Esta interpretación es congruente con las condiciones paleoambientales inferidas del estudio sedimentológico y de facies que indican que los sedimentos pliocenos se formaron en un ambiente de plataforma interna carbonatada donde abanicos deltaicos descargaban material terrígeno procedente de los relieves próximos (Aguirre, 2000).

En este contexto de alta energía, se observa una buena selección de tamaños de bioclastos en prácticamente todas las estaciones de muestreo; niveles 2 y 4 , localizados en la matriz calcarenítica, y niveles $3,6,7$ y 9, correspondientes a conglomerados fosilíferos y lags bioclásticos.

En el caso de los niveles inferiores, niveles 1a y 1c, correspondientes a facies interpretadas como depósitos litorales y abanico deltaico interno respectivamente (Aguirre, 2000), cabría esperar una selección de tamaños de bioclastos muy buena producida por destrucción y/o transporte selectivo. Sin embargo, la mala granoselección observada en estos niveles podría explicarse por la existencia de enterramientos esporádicos en zonas próximas a la paleocosta debido a los aportes procedentes de los abanicos deltaicos. De esta forma, restos de tamaños variables podrían quedar eventualmente enterrados de forma permanente. El hecho de que en estos niveles aparezcan algunos restos completos y articulados (Tabla 1) es congruente con esta interpretación.

En los niveles 5 y 8, concentraciones de Hippochae$t a$, también se observa una gran dispersión de tamaños de bioclastos. En ambos casos, la ausencia de granoclasifica-

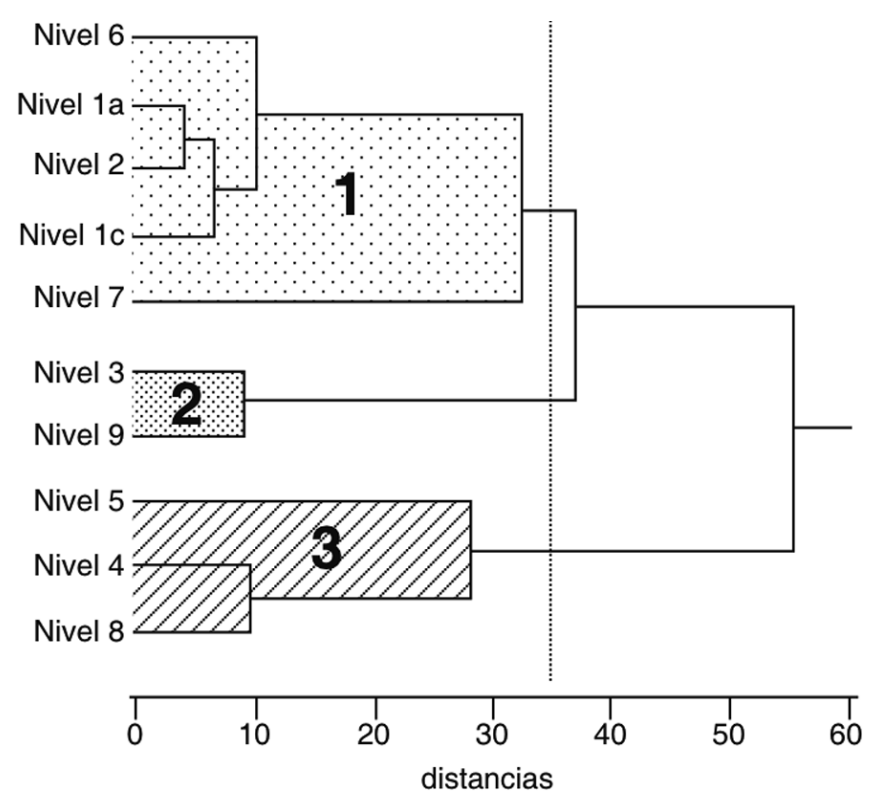

Figura 6. Dendrograma resultante del análisis cluster en modo $\mathrm{Q}$ realizado.

Resulting diagram from the Q-mode cluster analysis. 
ción se debe a la mezcla de conchas de gran talla de $\mathrm{Hi}$ ppochaeta, que pueden llegar a alcanzar tamaños de hasta $25 \mathrm{~cm}$ de diámetro máximo, con los restos bioclásticos que se encuentran en la matriz calcarenítica-calcirrudítica.

El hecho de que predominen los restos orientados con la concavidad hacia arriba en prácticamente todos los niveles estudiados (Fig. 5) puede estar relacionado bien con eventos de alta energía puntuales seguidos de enterramientos rápidos como las tormentas o bien con la actividad de organismos bioturbadores. En el primer caso, las tormentas remueven el sedimento del fondo marino y hacen que las conchas de los organismos se pongan en suspensión. Una vez que pasa el periodo de máxima energía los restos esqueléticos se redepositan sobre el fondo con la concavidad preferentemente hacia arriba. Así mismo, con la resaca de las tormentas, el sedimento se deposita rápidamente y puede enterrar de forma permanente los restos esqueléticos (Seilacher \& Aigner, 1991; Finnegan \& Droser, 2008). En el segundo caso, los organismos que se desplazan sobre el fondo marino, organismos infaunicos, epifaunicos o nectobentónicos, pueden removilizar los restos esqueléticos acumulados en el fondo y desplazarlos de su posición de equilibrio hidrodinámico (Kidwell \& Bosence, 1991; Parsons \& Brett, 1991). En los depósitos pliocenos de Almayate se encuentran evidencias a favor de ambos mecanismos. En este sentido, se reconocen niveles de tormentas y existen numerosas trazas de bioturbación de escape que distorsionan el sedimento y pueden reorientar las conchas (Aguirre, 2000).

Una evidencia a favor del efecto de las tormentas es la presencia de bivalvos apilados por su cara cóncava, ordenamiento stacking, observado especialmente en los niveles superiores de la secuencia pliocena (Figs. 4e-4g). Este tipo de biofábrica ha sido atribuido a dos procesos: a) deformación in situ debido a la acción de terremotos (Seilacher, 1991). En arenas y arcillas no consolidadas, en el momento del evento sísmico, parte del material más superficial es puesto en suspensión y redepositado lentamente desde la nube de sedimento. Por su parte, las conchas se acumulan de forma empaquetada (stacked) durante la liquefacción del sedimento producido por el seísmo (Seilacher, 1991). b) Flujos turbulentos de alta densidad producidos por tormentas (Middleton, 1967; Kidwell, 1991; Kidwell \& Holland, 1991). En los materiales estudiados, la existencia de muros erosivos, de una gradación vertical por tamaños y de amalgamaciones internas indican corrientes de tracción con potencial erosivo más o menos importante que corroborarían un origen por tormentas (Aguirre, 2000).

Otro rasgo tafonómico a considerar es el predominio de fósiles dispuestos perpendicularmente que se observa en la mayoría de las estaciones de muestreo (Fig. 5 y Tabla 1). Como Salazar-Jiménez et al. (1982) han puesto de manifiesto experimentalmente (ver también SchneiderStorz et al., 2008), en los subambientes de alta energía se obtiene un patrón de orientación similar al encontrado en los sedimentos pliocenos de Almayate. Esta explicación es congruente con el resto de los datos comentados hasta ahora. En las arenas gruesas de la parte basal de la sección, nivel 1a, cabe destacar un predominio de restos imbricados (Fig. 5), dispositivo que se produce en un fondo sometido a un continuo movimiento por tracción (Kidwell \& Holland, 1991).

\section{MODELOS DE TAFOFACIES}

La alta proporción de fragmentación, abrasión y desarticulación que caracteriza en general los materiales estudiados permite asignarlos al modelo de tafofacies de plataforma interna descrito en depósitos coetáneos de las cuencas de Almería-Níjar (Aguirre, 1996; Yesares-García \& Aguirre, 2004) y de Vejer de la Frontera (Cádiz; Rico et al., 2008). También es equivalente al Modelo I de tafofacies que Speyer \& Brett $(1986,1988,1991)$ definen en los depósitos del Devónico medio del estado de Nueva York. Estos modelos de tafofacies caracterizarían ambientes someros de alta energía y con una baja tasa de sedimentación que dejaría los restos esqueléticos expuestos durante prolongados períodos en la zona tafonómicamente activa antes de ser definitivamente enterrados. Según Smith \& Nelson (2003) estos serían los factores clave que controlan las tafofacies en plataformas carbonatadas templadas. Estos autores atribuyen la destrucción de los restos esqueléticos a la acción combinada de procesos físicos, biológicos y químicos (disolución temprana). En general, bajo estas condiciones, las propiedades tafonómicas que definen la tafofacies reflejan esencialmente las condiciones del fondo marino durante la sedimentación normal (background taphonomic processes de Speyer \& Brett, 1991).

Entre las propiedades tafonómicas que caracterizarían los modelos de tafofacies de plataforma interna o Modelo I están el predominio de restos orientados paralelamente a la estratificación y con la concavidad hacia abajo que es la posición hidrodinámicamente estable en ambientes energéticos (Speyer \& Brett, 1986, 1988, 1991; Aguirre, 1996; Yesares-García \& Aguirre, 2004; Rico et al., 2008). Sin embargo, en los niveles muestreados hay una proporción significativamente alta de restos orientados con la concavidad hacia arriba y dispuestos perpendicularmente con relación a la estratificación. Como se ha comentado anteriormente, la removilización episódica del sedimento debido a la acción de tormentas y a la actividad bioturbadora de los organismos podría explicar esa desviación en la orientación de la concavidad y la disposición con relación a la estratificación. En definitiva, estas propiedades tafonómicas estarían controladas por estos procesos episódicos (episodic taphonomic processes de Speyer \& Brett, 1991).

Si bien el conjunto de propiedades tafonómicas medidas permite asignar los sedimentos estudiados al modelo de tafofacies de plataforma interna, el análisis cluster diferencia tres grupos de muestras que comparten propiedades tafonómicas. Estas diferencias tafonómicas responden 
a las condiciones paleoambientales particulares en las que se formaron los diferentes niveles muestreados.

De las tres agrupaciones resultantes, el cluster 3 se diferencia claramente de los otros dos por el alto contenido en moldes frente a restos que conservan la mineralogía original de la concha (Fig. 5). Los moldes proceden, mayoritariamente, de conchas originalmente aragoníticas. Así mismo, las conchas de calcita presentes en estos niveles, casi exclusivamente valvas de Chlamys spp., muestran disolución total o parcial que se manifiesta porque las conchas sólo conservan alguna de las capas de la microestructura original y presentan una textura pulverulenta debido al efecto de la disolución. La presencia de moldes junto con la disolución total o parcial de conchas calcíticas implica unas condiciones ambientales contrarias. Por un lado, la formación de moldes requeriría de una precipitación temprana de carbonato cálcico, mientras que la disolución de las conchas calcíticas indicaría un medio químicamente muy agresivo opuesto a la precipitación (por ejemplo, Tucker, 1991; Canfield \& Raiswell, 1991).

Los procesos fosildiagenéticos tempranos en los carbonatos templados suelen ser destructivos ya que las aguas suelen estar poco saturadas o incluso infrasaturadas con respecto al carbonato cálcico (Alexandersson, 1978; Nelson et al., 1988b; Smith, 1988). Por tanto, las conchas de aragonito, fase metaestable del $\mathrm{CO}_{3} \mathrm{Ca}$, suelen sufrir disolución antes de la propia litificación del sedimento (Tucker, 1991; Hood \& Nelson, 1996; Nelson \& James, 2000; Smith \& Nelson, 2003; James et al., 2005). Esto conlleva un sesgo de conservación en las asociaciones fósiles (James et al., 2005; no obstante, ver Behrensmeyer et al., 2005 para una conclusión contraria en relación a las asociaciones fósiles post-paleozoicas) que, en última instancia, afecta a las interpretaciones a gran escala del propio registro fósil (Aguirre \& Jiménez, 1997; Cherns \& Wright, 2000, 2009). Sin embargo, bajo condiciones locales específicas se puede producir precipitación temprana de carbonato cálcico en las plataformas carbonatadas templadas (Reeckmann, 1988; Nelson \& James, 2000; Mutti \& Bernoulli, 2003; Caron et al., 2005). Nelson \& James (2000) reconocen cuatro tipos de precipitación temprana de $\mathrm{CO}_{3} \mathrm{Ca}$ en los carbonatos templados marinos cenozoicos del sur de Australia y Nueva Zelanda: a) Tipo 1, inmediatamente encima o debajo de una superficie de discontinuidad; b) Tipo 2, en el techo de ciclos submareales menores; c) Tipo 3, dentro de bioconstrucciones de ostras y/o briozoos; y, d) Tipo 4, en el interior de secuencias superpuestas de calcarenitas-calcirruditas con estratificaciones cruzadas. En el caso de los niveles con moldes del cluster 3 reconocido en los sedimentos estudiados en la Cuenca de Almayate, la cementación temprana sería equivalente al tipo 4 de Nelson \& James (2000). Por comparación con lo que ocurre actualmente en el nordeste de Australia (Keene \& Harris, 1995), la precipitación del $\mathrm{CO}_{3} \mathrm{Ca}$ en este contexto de alta energía estaría favorecida por el bombeo de agua marina por debajo de la interfase agua/sedimento en materiales muy permeables (Nelson \& James, 2000).

En el resto de niveles muestreados, si bien no se han reconocido moldes cabe pensar que muy posiblemente existieran organismos de concha aragonítica en las comunidades originales. De hecho, en las plataformas templadas del sur de Australia, James et al. (2005) estiman que entre el $50 \%$ y el $80 \%$ de los organismos que componen las comunidades que habitan en la plataforma presentan un esqueleto de aragonito. Es posible pensar, por tanto, que las conchas aragoníticas del resto de la sección estudiada se disolvieron antes de la litificación del sedimento, como suele ocurrir en estos ambientes templados, produciendo asociaciones de fósiles muy sesgadas.

La disolución de las conchas calcíticas, dado que se requiere de un medio químicamente muy agresivo y contrario a las condiciones para que se formen moldes, podría estar relacionada con procesos fosildiagenéticos tardíos. En este caso, la disolución pudo producirse por la acción de aguas meteóricas. Este hecho, probablemente, afectó a las propiedades tafonómicas observadas en los niveles donde se produjo disolución de calcita. Por un lado, la disolución provoca un debilitamiento de la estructura cristalina de las conchas, lo que potencia la fragmentación de los restos. Por otro lado, la disolución química puede producir resultados similares a los originados por la abrasión mecánica (Flessa \& Brown, 1983; Brett \& Baird, 1986; Davies et al., 1990; Parsons \& Brett, 1991; Glover \& Kidwell, 1993). En definitiva, la disolución genera y/o amplifica otros atributos tafonómicos producidos por procesos físicos, por lo que produce una señal tafonómica compuesta en el sentido de Speyer \& Brett (1991) que puede sesgar los resultados tafonómicos.

Por su parte, las otras dos agrupaciones del análisis cluster (Fig. 6) muestran propiedades tafonómicas similares aunque con algunas diferencias. Los niveles agrupados en el cluster 2 muestran un empaquetamiento denso con un alto porcentaje de fósiles por volumen de roca $(40 \%)$ y bajas tasas de interacciones orgánicas (encostramientos y bioperforaciones). Por el contrario, los niveles del cluster 1 muestran un empaquetamiento disperso con un porcentaje de fósiles por volumen de roca muy bajo $(10 \%)$ y altas proporciones de epifaunicos y de bioperforaciones. Los niveles del cluster 2 se interpretan como concentraciones residuales de conchas en la base de tempestitas. Debido a la alta energía de la tormenta, el fondo se excava localmente y el sedimento de menor tamaño es transportando mientras que los restos más gruesos se concentran en la base de los canales (formación de lags). Tras la tormenta, el material que se puso en suspensión se deposita enterrando los restos esqueléticos acumulados, originando la tempestita bioclástica (Finnegan \& Droser, 2008). Este enterramiento, a su vez, hace que los restos esqueléticos sufran bajas tasas de colonización y de bioperforaciones. Las propiedades tafonómicas que caracterizan estos niveles 
se corresponden con la tafofacies de niveles de tempestitas de Hendy et al. (2006) y Rico et al. (2008).

Los niveles del cluster 1, por el contrario, representan depósitos formados durante las condiciones normales de sedimentación. El hecho de que las conchas permanecieran durante prolongados periodos de tiempo expuestos sobre el fondo favoreció las colonizaciones y las bioperforaciones. Una alta incidencia de los organismos perforadores sobre las conchas, unido al efecto destructivo hidrodinámico, pudo incrementar la tasa de destrucción tafonómica.

El nivel 7, si bien está unido al cluster 1, lo hace a una gran distancia. A su vez, el cluster 1 se relaciona con el cluster 2 a una distancia relativamente corta con relación al nivel 7 (Fig. 6). En concreto, el nivel 7 se localiza en un conglomerado bioclástico con base erosiva que se interpreta como un depósito derivado de los abanicos deltaicos posteriormente retrabajado en la plataforma por algún fenómeno de tormenta (Aguirre, 2000). De esta forma, una tormenta removilizaría sedimento conglomerático junto con los restos bioclásticos acumulados en el fondo. El resultado final sería la mezcla de conglomerados y fósiles como depósitos residuales en la base de niveles canalizados. Según esta interpretación, el nivel 7 muestra propiedades tafonómicas intermedias entre los niveles del cluster 1 y del cluster 2, ya que los fósiles acumulados en la plataforma (con las propiedades tafonómicas que caracterizan el cluster 1) se mezclan y concentran en tempestitas (cuyas propiedades tafonómicas caracterizarían los niveles del cluster 2). Este hecho podría explicar que el nivel 7 se sitúe a una distancia intermedia entre el cluster $1 \mathrm{y}$ el cluster 2 .

\section{CONCLUSIONES}

1.- Los depósitos pliocenos que afloran en la Cuenca de Almayate son carbonatos templados caracterizados por la gran abundancia de bivalvos y briozoos. En este sentido, difieren del resto de depósitos coetáneos que afloran en la costa malagueña, esencialmente depósitos siliciclásticos.

2.- El estudio tafonómico de las asociaciones de fósiles sugiere unas condiciones de depósito de alta energía y un prolongado tiempo de exposición de los restos en la zona tafonómicamente activa: alta tasa de fragmentación, alto porcentaje de abrasión y abundancia de bioperforaciones. Estas propiedades tafonómicas, junto con la disolución temprana de las fases inestables de los carbonatos, caracterizan los procesos que ocurren durante los periodos de sedimentación normal en las plataformas de carbonatos templados.

3.- Procesos eventuales, como las tormentas, dejarían su impronta sedimentológica en forma de niveles de tempestitas. Se trata de concentraciones residuales de fósiles en la base de niveles canalizados. Las características tafonómicas de los restos esqueléticos concentrados en las tempestitas son una importante tasa de fragmentación y abrasión, una baja proporción de bioperforaciones y encostramientos, abundancia de restos orientados verticalmente y con la concavidad hacia arriba, y ordenamiento empaquetado de fósiles (stacking).

4.- Eventualmente, procesos de cementación temprana favorecieron la formación de moldes de organismos que presentaban conchas originalmente de aragonito. En los carbonatos templados domina la disolución. Sin embargo, bajo condiciones ambientales particulares se puede producir una cementación temprana. En ambientes de alta energía, donde se forman dunas submarinas, se puede producir precipitación temprana de carbonato cálcico como consecuencia de un bombeo de agua marina por debajo de la interfase agua/sedimento en un sedimento muy permeable.

5.- Un análisis cluster en modo Q permite diferenciar tres agrupaciones de muestras por sus características tafonómicas. Un grupo de muestras representan los depósitos afectados por los procesos destructivos que ocurren durante los procesos de sedimentación normal (background). Otro grupo de muestras representan niveles de tempestitas. El tercer grupo de muestras incluye aquéllas dominadas por la presencia de moldes sobre restos esqueléticos que conservan la mineralogía original de la concha.

\section{AGRADECIMIENTOS}

Queremos expresar nuestro agradecimiento a la Dra. Rosa Domènech y al Dr. Sixto Fernández-López por la revisión del manuscrito y sus comentarios para mejorar la calidad del mismo. Agradecemos también al Dr. Rodolfo Gozalo por su labor editorial. Este trabajo ha sido financiado por el Proyecto de Investigación CGL2007-60774/BTE del Ministerio de Educación y Ciencia y por el Grupo de Investigación RNM 013 de la Junta de Andalucía.

\section{BIBLIOGRAFÍA}

Aguirre, J. 1992. Evolución de las asociaciones fósiles del Plioceno marino de Cabo Roche (Cádiz). Revista Española de Paleontología, $\mathbf{n}^{\mathbf{0}}$ Extra., 3-10.

Aguirre, J. 1996. Modelo de tafofacies en el Plioceno inferior de la Cuenca de Almería-Níjar (SE de España). In: II Reunión de Tafonomía y Fosilización (Eds. G. MeléndezHevia, M.F. Blasco-Sancho \& I. Pérez-Urresti). Zaragoza, 11-18.

Aguirre, J. 2000. Evolución paleoambiental y análisis secuencial de los depósitos pliocenos de Almayate (Málaga, sur de España). Revista de la Sociedad Geológica de España, 13, 431-443.

Aguirre, J. \& Jiménez, A.P. 1997. Census assemblages in hard-bottom coastal communities; a case study from the Plio-Pleistocene Mediterranean. Palaios, 12, 598-608.

Aguirre, J. \& Jiménez, A.P. 1998. Fossil analogues of presentday Cladocora caespitosa coral banks: Sedimentary set- 
ting, dwelling community, and taphonomy (Late Pliocene, W Mediterranean). Coral Reefs, 17, 203-213.

Aguirre, J. \& Yesares-García, J. 2003. Tafonomía y análisis secuencial del Plioceno inferior en el sector NE de la Cuenca de Almería-Níjar (SE de España). Revista Española de Paleontología, 18, 61-82.

Aguirre, J., Cachão, M., Domènech, R., Lozano-Francisco, M.C., Martinell, J., Mayoral, E., Santos, A., Vera-Peláez, J.L. \& da Silva, C.M. 2005. Integrated biochronology of the Pliocene deposits of the Estepona basin (Málaga, S Spain). Palaeobiogeographic and palaeoceanographic implications. Revista Española de Paleontología, 20, 225-245.

Aguirre, J., Martín, J.M., Braga, J.C., Betzler, C., Berning, B. \& Buckeridge, J.S. 2008. Densely packed concentrations of sessile barnacles (Cirripedia: Sessilia) from the Early Pliocene of SE Spain. Facies, 54, 193-206.

Alexandersson, E.T. 1978. Destructive diagenesis of carbonate sediments in the eastern Skagerrak, North Sea. Geology, 6, 324-327.

Behrensmeyer, A.K., Fürsich, F.T., Gastaldo, R.A., Kidwell, S.M., Kosnik, M.A., Kowalewski, M., Plotnick, R.E., Rogers, R.R. \& Alroy, J. 2005. Are the most durable shelly taxa also the most common in the marine fossil record? Paleobiology, 31, 607-623.

Betzler, C., Brachert, T.C., Braga, J.C. \& Martín, J.M. 1997. Nearshore, temperate, carbonate depositional systems (lower Tortonian, Agua Amarga Basin, southern Spain): implications for carbonate sequence stratigraphy. Sedimentary Geology, 113, 27-53.

Betzler, C., Martín, J.M. \& Braga, J.C. 2000. Non-tropical carbonates related to rocky submarine cliffs (Miocene, Almería, southern Spain). Sedimentary Geology, 131, 51-65.

Braga, J.C., Martín, J.M. \& Wood, J.L. 2001. Submarine lobes and feeder channels of redeposited, temperate carbonate and mixed silicilastic-carbonate platform deposits (Vera Basin, Almería, southern Spain). Sedimentology, 48, 99-116.

Braga, J.C., Betzler, C., Martín, J.M. \& Aguirre, J. 2003. Spitplatform temperate carbonates: the origin of landwarddownlapping beds along a basin margin (Lower Pliocene, Carboneras Basin, SE Spain). Sedimentology, 50, 553-563.

Braga, J.C., Martín, J.M., Betzler, C. \& Aguirre, J. 2006. Models of temperate carbonate deposition in Neogene basins in SE Spain: a synthesis. In: Cool-Water Carbonates: Depositional Systems and Palaeoenvironmental Controls (eds. H.M. Pedley \& G. Carannante). Geological Society of London, Special Publication, 225, 121-135.

Braga, J.C., Martín, J.M., Aguirre, J., Baird, C.D., Grunnaleite, I., Jensen, N.B., Puga-Bernabéu, A., Saelen, G. \& Talbot, M.R. 2010. Middle-Miocene (Serravallian) temperate carbonates in a seaway connecting the Atlantic Ocean and the Mediterranean Sea (North Betic Strait, S Spain). Sedimentary Geology, 255, 19-33.

Brett, C.E. \& Baird, G.C. 1986. Comparative taphonomy: a key to paleoenvironmental interpretation based on fossil preservation. Palaios, 1, 207-227.
Canfield, D.E. \& Raiswell, R. 1991. Carbonate precipitation and dissolution: Its relevance to fossil preservation. In: Taphonomy. Releasing the Data Locked in the Fossil Record (eds. P.A. Allison \& D.E.G. Briggs). Plenum Press, New York, 411-453.

Carannante, G., Esteban, M., Milliman, J.D. \& Simone, L. 1988. Carbonate lithofacies as paleolatitude indicators: problems and limitations. Sedimentary Geology, 60, 333-346.

Caron, V., Nelson, C.S. \& Kamp, P.J.J. 2005. Sequence stratigraphic context of syndepositional diagenesis in cool-water carbonates: Pliocene limestone, New Zealand. Journal of Sedimentary Research, 75, 231-250.

Chave, K.E. 1967. Recent carbonate sediments: an unconventional view. Journal of Geological Education, 19, 200-204.

Cherns, L. \& Wright, V.P. 2000. Missing molluscs as evidence of large-scale, early skeletal aragonite dissolution in a Silurian sea. Geology, 28, 791-794.

Cherns, L. \& Wright, V.P. 2009. Quantifying the impact of early diagenetic aragonite dissolution on the fossil record. Palaios, 24, 756-771.

Davies, D.J., Staff, G.M., Callender, W.R. \& Powell, E.N. 1990. Description of a quantitative approach to taphonomy and taphofacies analysis: All dead things are not created equal. In: Paleocommunity Temporal Dynamics: The Long-Term Development of Multispecies Assemblages (ed. W. Miller III). Paleontological Special Publication, 5, 328-350.

Davies, G.R. 1970. Carbonate-bank sedimentation, eastern Shark-Bay, western Australia. American Association Petroleum Geologists, Memoir, 13, 85-168.

Finnegan, S. \& Droser, M.L. 2008. Reworking diversity: Effects of storm deposition on evenness and sampled richness, Ordovician of the basin and range, Utah and Nevada, USA. Palaios, 23, 87-96.

Flessa, K.W. \& Brown, T.J. 1983. Selective solution of macroinvertebrate calcareous hard parts: A laboratory study. Lethaia, 16, 193-205.

Ginsburg, R.N. 1956. Environmental relationship of grain size and constituent particles in some south Florida carbonate sediments. American Association Petroleum Geologists Bulletin, 40, 2384-2427.

Glover, C.P. \& Kidwell, S.M. 1993. Influence of organic matrix on the post-mortem destruction of molluscan shells. Journal of Geology, 101, 729-747.

Guerra-Merchán, A. \& Serrano, F. 1993. Análisis estratigráfico de los materiales neógeno-cuaternarios de la región de Nerja. In: Geología de la Cueva de Nerja (ed. F. Carrasco). Patronato de la Cueva de Nerja, Trabajos sobre la Cueva de Nerja, 3, 55-90.

Hallock, P. 1981. Production of carbonate sediment by selected large foraminifera on two Pacific coral reefs. Journal of Sedimentary Petrology, 51, 467-474.

Hayton, S., Nelson, C.S. \& Hood, S.D. 1995. A skeletal assemblage classification system for non-tropical carbonate deposits based on New Zealand Cenozoic limestones. Sedimentary Geology, 100, 123-141. 
Hendy, A.J.W., Kamp, P.J.J. \& Vonk, A.J. 2006. Cool-water shell bed taphofacies from Miocene-Pliocene shelf sequences in New Zealand: utility of taphofacies in sequence stratigraphy analysis. In: Cool-Water Carbonates: Depositional Systems and Palaeoenvironmental Controls (eds. H.M. Pedley \& G. Carannante). Geological Society of London, Special Publication, 225, 283-305.

Hood, S.D. \& Nelson, C.S. 1996. Cementation scenarios for New Zealand Cenozoic nontropical limestones. New Zealand Journal of Geology and Geophysics, 39, 109-122.

James, N.P. 1997. The cool-water carbonate depositional realm. In: Cool-Water Carbonates (eds. N.P. James \& A.D. Clarck). SEMP Special Publication, 56, 1-20.

James, N.P. \& Clarke, A.D. (eds.) 1997. Cool-Water Carbonates. SEMP Special Publication, 56, 440 pp.

James, N.P., Yvonne, B. \& Kyser, T.K. 2005. Where has all the aragonite gone? Mineralogy of Holocene neritic cool-water carbonates, southern Australia. Journal of Sedimentary Research, 75, 454-463.

Keene, J.B. \& Harris, P.T. 1995. Submarine cementation in tide-generated bioclastic sand dunes: epicontinental sea-way, Torres Strait, north east Australia. International Sedimentology, Special Publication, 24, 225-236.

Kidwell, S.M. 1991. Condensed deposits in siliciclastic sequences: expected and observed features. In: Cycles and Events in Stratigraphy (eds. G. Einsele, W. Ricken \& A. Seilacher). Springer-Verlag, Berlin, 682-695.

Kidwell, S.M. \& Bosence, D.W.J. 1991. Taphonomy and time-averaging of marine shelly fauna. In: Taphonomy. Releasing the Data Locked in the Fossil Record (eds. P.A. Allison \& D.E.G. Briggs). Plenum Press, New York, 115-209.

Kidwell, S.M. \& Holland, S.M. 1991. Field description of coarse bioclastic fabrics. Palaios, 6, 426-434.

Kidwell, S.M., Fürsich, F.T. \& Aigner, T. 1986. Conceptual framework for the analysis and classification of fossil concentrations. Palaios, 1, 228-238.

Lees, A. \& Buller, A.T. 1972. Modern temperate-water and warm-water shelf carbonate sediments contrasted. Marine Geology, 13, 67-73.

Martín, J.M., Braga, J.C., Betzler, C. \& Brachert, T.C. 1996. Sedimentary model and high-frequency cyclicity in a Mediterranean, shallow-shelf, temperate-carbonate environment (uppermost Miocene, Agua Amarga Basin, Southern Spain). Sedimentology, 43, 263-277.

Martín, J.M., Braga, J.C., Aguirre, J. \& Betzler, C. 2004. Contrasting models of temperate-carbonate sedimentation in a Pliocene, small Mediterranean-Sea embayment (Carboneras Basin, SE Spain). Journal of the Geological Society of London, 161, 387-399.

Martín, J.M., Braga, J.C., Sánchez-Almazo, I.M. \& Aguirre, J. 2009a. Temperate and tropical carbonate-sedimentation episodes in the Neogene Betic basins (S Spain) linked to climatic oscillations and changes in the AtlanticMediterranean connections. Constraints with isotopic data. In: Oligocene-Miocene carbonate systems (eds. C.
Betzler, M. Mutti, \& W.E. Piller). IAS, Special Publication, 42, 49-70.

Martín, J.M., Braga, J.C., Aguirre J. \& Puga-Bernabéu, A. 2009b. History and evolution of the North-Betic Strait (Prebetic Zone, Betic Cordillera): A narrow, early Tortonian, tidal-dominated, Atlantic-Mediterranean marine passage. Sedimentary Geology, 216, 80-90.

Mayoral, E. \& Rodríguez-Vidal, J. 1994. Aspectos morfosedimentarios de la transgresión pliocena en Almayate (Málaga). Geogaceta, 16, 110-113.

Meldahl, K.H. \& Flessa, K.W. 1990. Taphonomic pathways and comparative biofacies and taphofacies in a recent intertidal/shallow shelf environment. Lethaia, 23, 43-60.

Middleton, G.V. 1967. The orientation of concave-convex particles deposited from experimental turbidity currents. Journal of Sedimentary Petrology, 37, 229-232.

Mutti, M. \& Bernoulli, D. 2003. Early marine lithification and hardground development on a Miocene ramp (Maiella, Italy): key surfaces to track changes in trophic resources in nontropical carbonate settings. Journal of Sedimentary Research, 73, 296-308.

Mutti, M. \& Hallock, P. 2003. Carbonate systems along nutrients and temperature gradients: some sedimentological and geochemical constraints. International Journal of Earth Sciences, 92, 465-475

Mutti, M., Bernoulli, D. \& Stille, P. 1997. Temperate carbonate platform drowning linked to Miocene oceanographic events: Maiella platform margin, Italy. Terra Nova, 9, 122-125.

Nelson, C.S. (ed.) 1988. Non-tropical shelf carbonates modern and ancient. Sedimentary Geology, 60, 367 pp.

Nelson, C.S. \& James, N.P. 2000. Marine cements in midTertiary cool-water shelf limestones of New Zealand and southern Australia. Sedimentology, 47, 609-629.

Nelson, C.S., Keane, S.L. \& Head, P.S. 1988a. Non-tropical carbonate deposits on the modern New Zealand shelf. Sedimentary Geology, 60, 71-94.

Nelson, C.S., Harris, G.J. \& Young, H.R. 1988b. Burialdominated cementation in non-tropical carbonates of the Oligocene Te Kuiti Group, New Zealand. Sedimentary Geology, 60, 233-250.

Parsons, K.M. \& Brett, C.E. 1991. Taphonomic processes and biases in modern marine environments: an actualistic perspective on fossil assemblage preservation. In: The Processes of Fossilization (ed. S.K. Donovan). Belhaven Press, London, 22-65.

Pedley, H.M. \& Carannante, G. (eds.) 2006. Cool-Water Carbonates: Depositional Systems and Palaeoenvironmental Controls. Geological Society of London, Special Publication, 255, 373 pp.

Pomar, L., Brandano, M. \& Westphal, H. 2004. Environmental factors influencing skeletal grain sediment associations: a critical review of Miocene examples from the western Mediterranean. Sedimentology, 51, 627-651.

Puga-Bernabéu, A., Martín, J.M. \& Braga, J.C. 2007. Tsunami-related deposits in temperate carbonate ramps, 
Sorbas basin, southern Spain. Sedimentary Geology, 199, 107-127.

Puga-Bernabéu, A., Martín, J.M. \& Braga, J.C. 2008. Sedimentary processes in a submarine canyon excavated into a temperate-carbonate ramp (Granada Basin, southern Spain). Sedimentology, 55, 1449-1466.

Puga-Bernabéu, A., Martín, J.M. \& Braga, J.C. 2010. Downslope-migrating sandwaves and platform-margin clinoforms in a current-dominated, distally steepened temperate-carbonate ramp (Guadix Basin, Southern Spain). Sedimentology, 57, 293-311.

Purdy, E.G. 1963. Recent carbonate facies of the Great Bahama Bank, II. Sedimentary facies. Journal of Geology, 71, 472-497.

Reeckmann, S.A. 1988. Diagenetic alterations in temperate shelf carbonates from southern Australia. Sedimentary Geology, 60, 209-219.

Rico, A., Aguirre, J. \& González-Delgado, J.A. 2008. Taphonomy and taphofacies models of the Pliocene deposits of Vejer de la Frontera (Cádiz, SW Spain). Geobios, 41, 543-558.

Salazar-Jiménez, A., Frey, R.W. \& Howard, J. 1982. Concavity orientations of bivalve shells in estuarine and nearshore shelfs sediments, Georgia. Journal of Sedimentary Petrology, 52, 565-586.

Sánchez-Almazo, I.M., Spiro, B., Braga, J.C. \& Martín, J.M. 2001. Constraints of stable isotope signatures on the depositional palaeoenvironments of upper Miocene reef and temperate carbonates in the Sorbas basin, SE Spain. Palaeogeography, Palaeoclimatology, Palaeoecology, 175, 153-172.

Schneider-Storz, B., Nebelsik, J.H., Wehrmann, A. \& Federolf, C.M.J. 2008. Comparative taphonomy of three bivalve species from a mass shell accumulation in the intertidal regime of North Sea tidal flats. Facies, 54, 461-478.

Seilacher, A. 1991. Events and their signatures. An overview. In: Cycles and Events in Stratigraphy (eds. G. Einsele, W. Ricken, \& A. Seilacher). Springer-Verlag, Berlin, 283-297.

Seilacher, A. \& Aigner, T. 1991. Storm deposition at the bed, facies and basin scale: The geologic perspective. In: Cycles and Events in Stratigraphy (eds. G. Einsele, W. Ricken, \& A. Seilacher). Springer-Verlag, Berlin, 249-267.
Serrano, F. 1993. Generalidades sobre la geología de la provincia de Málaga. IX Jornadas de Paleontología, 16-27.

Shinn, E.A., Lloyd, R.M., \& Ginsburg, R.N. 1969. Anatomy of a modern carbonate tidal flat, Andros Island, Bahamas. Journal of Sedimentary Petrology, 39, 1202-1228.

Smith, A.M. 1988. Preliminary steps toward formation of a generalized budget from cold-water carbonates. Sedimentary Geology, 60, 323-331.

Smith, A.M. \& Nelson, C.S. 2003. Effects of early sea-floor processes on the taphonomy of temperate shelf skeletal carbonate deposits. Earth-Science Reviews, 63, 1-31.

Speyer, S.E. \& Brett, C.E. 1986. Trilobite taphonomy and middle Devonian taphofacies. Palaios, 1, 312-327.

Speyer, S.E. \& Brett, C.E. 1988. Taphofacies models for epeiric sea environments: Middle Paleozoic examples. Palaeogeography, Palaeoclimatology, Palaeoecology, 63, 225-262.

Speyer, S.E. \& Brett, C.E. 1991. Taphofacies controls: background and episodic processes in fossil assemblage preservation. In: Taphonomy. Releasing the Data Locked in the Fossil Record (eds. P.A. Allison \& D.E.G. Briggs). Plenum Press, New York, 501-545.

Tucker, M.E. 1991. The diagenesis of fossils. In: The Processes of Fossilization (ed. S.K. Donovan). Belhaven Press, London, 84-104.

Tucker, M.E. \& Wright, V.P. 1992. Carbonate Sedimentology. Blackwell Scientific Publications, Oxford, 482 pp

Yesares-García, J. \& Aguirre, J. 2002. A methodological procedure for taphonomic analysis and taphofacies models. An experimental approach and a case study. In: Current Topics on Taphonomy and Fossilization (eds. M. De Renzi, M.V. Pardo-Alonso, M. Belinchón, E. Peñalver, P. Montoya \& A. Márquez-Aliaga). Ayuntamiento de Valencia, 435-443.

Yesares-García, J. \& Aguirre, J. 2004. Quantitative taphonomic analysis and taphofacies in lower Pliocene temperate carbonate-siliciclastic mixed platform deposits (AlmeríaNíjar basin, SE Spain). Palaeogeography, Palaeoclimatology, Palaeoecology, 207, 83-103.

Manuscrito recibido: 29 de Julio, 2010 Manuscrito aceptado: 25 de Noviembre, 2010 